\title{
TITLE: METHODS OF 3D DATA APPLICATIONS TO INFORM DESIGN DECISIONS FOR PHYSICAL COMFORT
}

\author{
AUTHORS \\ Stavros - Konstantinos Stavrakos \\ Division of Technology and Innovation Management \\ Department of Management Engineering \\ Technical University of Denmark \\ skost@man.dtu.dk \\ Tel: 004526130103 \\ Saeema Ahmed-Kristensen \\ Division of Technology and Innovation Management \\ Department of Management Engineering \\ Technical University of Denmark \\ $\underline{\text { sakr@dtu.dk }}$
}

\begin{abstract}
Many everyday tasks require physical contact with products, such as helmets, headsets, etc. There is a drive for comfortable products that fit the task and the human body, especially in the industry of external ear worn products. Past research on anthropometry stresses that positive comfort is enhanced when there is sufficient knowledge of human factors. The majority of these studies focus only on the acquirement and presentation of anthropometric data. This paper examines the incorporation of anthropometry in the design of external-ear devices. The aim of this paper is to provide different methods to support design applications (ways of analysis) of 3-dimensional head and ear data with a focus on external ear products. 200 persons representing the Danish population were scanned. The 3-d data was collected, refined and analysed. A matrix containing 29 new ear dimensions was generated. An analysis of the dataset through understanding of human and product geometry and methods of visualisation of the dataset generated an additional number of 9 advanced dimensions. The paper finally presents all phases of the analysis of the 3D data in the form of a methodological framework. The paper contributes with, in addition to the methodological framework, techniques to extract data based on a mathematical and product understanding, visualisation of data and manual extractions and how the data can be used to define archetypes for focus groups and other qualitative assessments. In their endeavour to develop successful and comfortable products designers should focus more on fitting the task into the human by benchmarking human dimensions against product data.
\end{abstract}

Keywords: ergonomics, design for interaction 
Comfort is an important consideration when designing products, especially those in physical contact with the user e.g. headsets or chairs. Industry aims to create comfortable products that fit the task and the human body in order to stay ahead of competition. There is a drive for the design of products which contribute to health and well-being, especially in the industry of external ear worn products, like headphones and Bluetooth headsets. Moreover, several types of external ear products are currently designed to be used in athletic activities, whilst running or cycling, for example. This increases the demand for better fitting products with low pressure levels. A challenge for design engineers and comfort specialists working at the early stages of the development of such products is to define a set of human factors in order to achieve high physical comfort. Defining these factors will enable designers to better predict physical factors of comfort such as good fit in the ear (in the case of wearing a Bluetooth device).

Past literature on ergonomics and human factors has provided anthropometric data regarding most areas of the human body. [Hibaru et al. 1994], [Jung and Jung, 2001], [Parcells et al. 1999] and a few ergonomists have attempted to make use of existing human datasets to inform design decisions. Past research on anthropometry stresses that comfort is achieved when there is sufficient knowledge of human factors. Comfort for consumer products is influenced by good fit and alliance between product dimensions and human dimensions. However, current databases for anthropometric ear data, e.g. Peoplesize Software [Peoplesize, 2008] lack advanced data regarding areas on the ear which are difficult to measure manually or require the use of 3D systems (e.g. back ear data). In addition, there seems to be limited literature in regards to an advanced mathematical analysis of the human dimensions which these papers provide. Hence, research is scarce in regards to the issuing of design guidelines to create a link between human dimensions and product dimensions for this context.

This paper examines the incorporation of anthropometry in the design of external-ear devices. Within this context, the aim of this paper is to provide different methods to support design by various applications (ways of analysis) of 3-dimensional head and ear data with a focus on external ear products based on the analysis of a 3-dimensional data set. The main aims of this paper is to investigate how human dimensions can be benchmarked against product dimensions with the aim to support product design and how product dimensions can be predicted through qualitative methods of visualisation of human data.

The paper consists of three parts: (1) first it reviews the existing literature, (2) then the hypothesis and the research methodology are presented and data analysis techniques are discussed and (3) this is followed by the discussion of the findings and limitations of the study. The paper concludes with a discussion of implications and directions for further research. 
This section presents the scientific background for this paper. Comfort definitions are introduced followed by past studies on influential factors of physical comfort; past studies on collection of 2- dimensional and 3 - dimensional anthropometric datasets with a focus on external ear data and; previous studies which develop methods to create links between human dimensions and product dimensions.

\subsection{Definitions of comfort - Comfort in the literature}

A debate lies in the body of scientific literature, regarding a commonly accepted definition of comfort. Slater [1987] defines comfort as a pleasant state of physiological, psychological and physical harmony between a human being and the environment. Richards [1980] states that comfort is the state of mind that involves a sense of subjective well-being in reaction to an environment or a situation. Vink [2005] focuses on the subjective view of comfort by stating that in the context of air flights "Comfort is a subjective experience. For Passenger 1 on a long distance flight, back discomfort is of great importance. Passenger 2 wants a reduction in noise and Passenger 3 needs more space." According to Vink (2005), comfort is defined as (1) a construct subjectively defined, (2) it is a reaction to the environment and (3) it is affected by factors of various natures linking to the participant (physical, psychological and physiological). This paper focuses primarily upon the physical properties of comfort i.e., the effect of the physical interaction between a human and a product over limited interaction time (the observed interaction lasts for 3-5 minutes).

\subsection{Physical loading, an influencing factor of comfort}

Among the many comfort studies which link discomfort to physical loading, Kee et al. [2012] investigated the relationships between the external load on human body (subjective measures of discomfort) and objective measures related to the assessment of postural stresses based on literature survey. Objective measures included posture holding time, Maximum Holding Time (MHT), torque at joints, Lifting Index (LI) and compressive force (CF) at L5/S1. The major relationships identified in this literature survey were the following: 1) postural discomfort linearly increased with increasing holding time, and holding force, 2) whole body discomfort was inversely linearly proportional to the MHT, 3) body-part discomfort was related to objective measures such as torque at the relevant joint and 4) discomfort was strongly linearly related to LIs and CFs, and Thus, discomfort might be used as a measure for quantifying postural stresses.

In a similar context, Zenk et al. [2012] conducted an objective assessment approach which evaluates the concept of "optimal load distribution", based on the identification of a close relationship between the pressure on the seat and the discomfort felt by the person sitting. The results indicate that in the seat position with the pressure distribution corresponding to the most comfortable posture the pressure in the intervertebral disc is lowest.

Hence, if the physical load forced on the user is kept down to low levels and the exposure of users to the load lasts for a considerable amount of time then their response can relate to 
objective measures. There is a strong connection between discomfort and physical dimensions, such as postural stress.

\subsection{Past research on collection of 2-d and 3-d human data with focus on ear data and improving design.}

Anthropometry is considered the very ergonomic core of any attempt to resolve the dilemma of fitting the tasks to the human [Sanders \& McCormick, 1993]. In regards to external ear products such as bluetooth headsets and headphones, good fit is a crucial physical factor of comfort to ensure the success of these products. Designers require anthropometric data to identify human factors and inform design decisions with respect to external ear devices. Current approaches are restricted in the presentation of anthropometric data only. The collection of ear data includes the use of various measurement instruments. Jung and Jung [2001] provided anthropometric dimensions of ears of Korean subjects using digital calipers. Other methods suggest the use of simple geometric calculations to acquire dimensions from a $2 \mathrm{D}$ photograph by setting reference points before taking the photographs. In regards to data collection of other body parts, such as head and legs data, other, relatively noninvasive, 3D imaging techniques are applied. These include various forms of stereophotogrammetry [Weinberg, et al. 2006], topography techniques [Ghoddousi, et al. 2006] and surface scanning technologies [Hennessy, et al. 2002]. In their paper, Stavrakos \& Ahmed-Kristensen [2012] compared three methods of collection of ear data (with a digital caliper, photogrammetrtic method and 3D scanning method), in terms of cost, time, accuracy and comfort and concluded that the 3D scanning method is the most costly yet the most accurate of all.

In other disciplines such as seating design, design engineers have attempted to design desks and chairs based on anthropometric data [Hibaru et al. 1994 ; Parcells et al. 1999]. Parcells et al. [1999] studied the mismatch between furniture and students' dimensions by measuring anthropometric characteristics of American children aged 11-13 years and the dimensions of their classrooms' desks and chairs, reporting that only $18.9 \%$ of students could find an appropriate match [Gouvali, et al. 2005]. Other studies provide detailed anthropometric data and some of them also offer recommendations for design [Klamklaya, 2008]. In their study, Stavrakos \& Ahmed-Kristensen et al. [2014], presented a validated methodological framework of how to generate archetypes from an anthropometric dataset of ears measured manually with a Vernier caliper, in order to build test panels for the execution of more reliable comfort studies.

The majority of these studies focus on the acquirement of anthropometric data, and this is limited to physical match. The anthropometric dimensions presented in these papers are not further analysed from a mathematical point of view to acquire a more advanced knowledge of the human geometry. Furthermore, no studies were found that propose methodologies to predict human factors with the attempt to improve physical comfort factors in product design. In particular in the ear industry, there appears to be a gap in the definition of a validated methodological framework to link ear anthropometry to design.

\subsection{Research Questions and Hypothesis}

This section presents the main research question (RQ) and hypothesis of the research presented. The main research question was: 
How can 3-D human data support the design of products - in this case, external, ear products for short term human - product interactions?

The set of hypothesis linked to the research question are presented here:

- (H1) By meaningful manipulations of 3-D human data, it is possible to benchmark human dimensions against product dimensions with the aim to support product design, and

- $\quad(\mathrm{H} 2)$ It is possible to predict product dimensions through qualitative methods of visualisation of human data.

In addition to the hypothesis presented, in this paper, the archetype methodology by Stavrakos and Ahmed-Kristensen et al. [2014] was reproduced using a 3Dimensional dataset. Hence, the archetype methodology will only be mentioned briefly in the Methods section.

3.

METHODS

The methodological framework of the various manipulations of 3-D data is presented in this section. As shown in Figure 1, the methodology consists of three main phases; (1) the Early Product Understanding phase; (2) the 3Dimensional Data collection phase and; (3) the various Applications of 3Dimensional Data phase. These phases, and the various steps they consist of, are described below and are summarised in Figure 1.

Figure 1.

\subsection{Early Product Understanding}

This paper investigates short-term interactions (2-3 minutes) between humans and external ear worn products, such as Bluetooth headsets, headphones, etc. Initially, product categories are created based on wearing style and fit. Five categories of external-ear worn products were defined (see Figure 2) for this study. At this stage, there was also the possibility of defining critical product components that touch upon critical areas of the human body. The interfaces would require a different treatment from a data collection point of view, such as the ear gel component in the "in the ear headsets" product category, which touches upon the in-the-ear area. However, this paper does not focus on product components, as the main anthropometric area which this paper focuses on is the external ear area. The researchers of this paper aimed to eliminate the possibility of having to treat in-the-ear data which would require different scanning techniques. The products mentioned in this paper are objects seen as one object with stabile physical properties.

Figure 2.

In the next step, critical areas were defined on the human surface. Critical areas were defined as the areas where a product is in physical contact to the human body.

Figure 3.

Once these areas were defined on the human head and ears, critical human dimensions were defined to describe as accurately as possible the critical area from a human-product 
interaction point of view, rather than a geometrical point of view, e.g. the ear to ear - over the head top dimension. The associations between critical product areas and the determination of critical human dimensions, for product families 1 and 3 can be seen in figure 3 .

Once the associations between critical areas of human-product interaction and human dimensions were completed, the authors issued a table listing all critical human dimensions to be collected in order to achieve product and human body understanding. In total, 25 linear and non-linear human dimensions were identified in respect to the 5 product categories of earworn products.

\subsection{3-Dimensional data collection}

Once the critical human dimensions have been determined, head and ear data was collected. The various steps of this process (see Figure 1) comprise of (1) the selection of the population, (2) the definition of a representative user sample, and (3) the scanning of the population. Table 1 also shows the demographics of the data collection of this study and the measurements performed on the 3D head scans to collect all critical human dimensions.

Table 1.

To ensure that the user sample was representative, a population with homogenous, ethnographic properties needs to be selected. The population of a country, which in this instance was Denmark, was selected for the execution of this study. To ensure that the user sample chosen to represent Denmark's population -which reaches to apx. 5,500,000 personsis representative, the statistical sample size of Denmark's population was calculated with the use of the following mathematical equation:

\section{Necessary Sample Size $=(Z \text {-score })^{2} * \operatorname{StdDev}^{*}(1-\mathrm{StdDev}) /(\text { margin of error })^{2}$}

In the case of Denmark, the sample size required is 196 people for a confidence level of $95 \%$, confidence interval $=7$ and population size $=5,500,00$ people. This means that if apx. 200 persons are randomly selected out of the 5,500,000, these can statistically represent sufficiently the population at hand from an anthropometric point of view. Hence, a randomized sample of 200 Danish people (100 men, 100 women) was chosen with ages ranging from 22 to 67 years to match the requirements of the calculated sample size for the scanning of the persons.

The steps of the scanning process are briefly depicted in figure 4. The 196 persons were scanned using a system consisting of one projector and two cameras which were both calibrated. A structured design was employed where patterns are projected onto the object's surface, pixel values were assigned by calculating the exact distance between points on the object's surface and the focal plane of the camera. In this system all visible points were calculated simultaneously in a single capture in a few seconds. Once the scanning took place the two images produced by the two cameras were merged with the help of a 3D modeling software. The outcome of this was a single 3D model.

Figure 4. 
The 196 3D Models were later processed using a 3d imaging software (G.O.M. Inspect 2.0). The purpose of this iteration was to refine critical areas which were hard to scan on site, such as areas behind the ears and human surface covered with hair. The G.O.M. imaging software allowed these areas to be restored by making use of a predictive algorithm that generated the missing areas by using the data of neighbouring human surface.

\subsection{Applications of 3-Dimensional Data}

The various steps of this process can be seen in Figure 1. Once the scanning of the participants and the refinement of the scans was complete, a dataset of 196 human head scans was available. Three main manipulations were identified and performed in this paper. They are the Basic and In-Depth benchmarking between Products and Human Factors, the retrieval of critical dimensions through Qualitative Methods of Data Analysis (e.g. Visualisations of 3D-Data), and the Creation of Focus Groups by utilising the archetype methodology described in depth in Stavrakos \& Ahmed-Kristensen [2014] and summarised for this paper in section 3.6.

\subsection{Basic and In-Depth Benchmarking between Products and Human Factors}

Regarding the basic benchmarking, the main step of this process (see Figure 1) concerns the selection of reference points and generation of a matrix of critical, linear human dimensions. In the basic benchmarking, the matrix of the associations between critical product areas and human dimensions was used (See figure 3). As stated earlier, 25 linear and non-linear human dimensions were generated from the matrix. The 25 dimensions were identified on all 196 head scans by generating reference points for the linear dimensions (starting point - ending point) and by drawing the respective curves to reproduce the non-linear ones. After all reference points were applied, the dimensions between the starting and ending point for each one of the linear dimensions were calculated, as well as the non-linear ones (see figure 5). At this stage, 4,900 measurements were retrieved manually from the imaging software. All activities were performed in G.O.M. Inspect. The measurements were inserted into a matrix and several statistical values were calculated, such as, minimum (MIN), maximum (MAX), median (MN) and average (AVE) values. Furthermore, tables of percentiles and frequency diagrams were calculated for all dimensions. These are presented in the results section.

Figure 5.

The in-depth benchmarking (see Figure 1) comprises of (1) a mathematical modelling of products, (2) selection of critical angles and through-the-mesh dimensions and (3) application of geometrical elements on the 196 scans. Considerations were made regarding the generation of additional human dimensions which could be retrieved by working through the mesh of points of the 3D scans, compared to the 25 dimensions which were identified on the surface of the human heads. For this purpose, 5 additional, through-the-mesh dimensions were defined. These can be seen in figure 6. The first two dimensions, Back Canal Top and Back Canal Bottom connect the top and bottom point of the ear canal to the middle point behind the ear. These dimensions are useful for the design of ear-product categories 4 and 5 (see figure 2), which covers ear products worn behind the ear and products that include ear hooks. 
Figure 6.

Calculating through-the-mesh dimensions, offered the possibility of generating three crucial dimensions for the design of headphones and products worn around the head. Figure 6 presents head height, head breadth top and head breadth bottom, which can inform the design of product categories 1 and 2, i.e., headphones over and around the ears, respectively. Two more dimensions were also added to support product categories 1 and 2, i.e., the design of headphones. These were angles $\varphi 1$ and $\varphi 2$, as shown in figure 7 . The angle $\varphi 1$ is calculated as the angle formed (see Figure 7) between segment (BC) and the height of the trapeze (ABCD), which, in this case, is segment L3. Segments (AB) and (DC), which are contained in the trapeze, are the human dimensions Head Breadth Top and Head Breadth Bottom, respectively, which were identified earlier (see figure 6):

$$
(\tan \varphi 1)^{-1}=((\mathrm{AB})-(\mathrm{DC})) / \mathrm{L} 3
$$

For the calculation of $\varphi 2$, two planes were formed. Plane A was formed by the two ear connection points top and bottom, and point $\mathrm{F}$, as shown in figure 7. Plane B was formed by the same ear connection points and the middle point along back circumference, i.e., the behind the ear middle point, as shown in figure 6.

\section{Figure 7.}

The angle $\varphi 2$ was calculated in G.O.M. Inspect as the angle between planes A and B for both left and right ear. Both angles were calculated for all 196 headscans. In total, 9 more dimensions were added to the list of critical human dimensions (Back Ear Canal Top and Bottom for both right and left ear, Head height, Head Breadth Top, Head Breadth Bottom and angles $\varphi 1$ and $\varphi 2$ ), i.e., 1,764 additional measurements were executed.

\subsection{Qualitative methods of data analysis - Visualisations of data}

The visualisation of data (see Figure 1) comprises of (1) the selection of reference points and curved lines, (2) overlay of geometry on the 196 scans, (3) and the calculation of product dimensions on the final image of the superimposed curves. An additional way to manipulate $3 \mathrm{D}$ data is to inform designers on the shape of product components and to generate human dimensions after a qualitative understanding of human geometry. In this paper, additional human dimensions were generated through visualisation of 3D human data. Figure 7 shows a bluetooth headset worn behind the ear. Initially two interfaces were identified, the one resting over the ear (green colour) and the other (red colour) resting behind the ear. An advanced knowledge of the area behind the ear is needed to inform the dimensions of the back product component.

Additionally, a joint movement, in the position where point A lies in figure 7, was identified with 3 degrees of freedom $(\mathrm{dF}=3)$. The mechanical model of the product components indicates that the back component rotates around point A, which lies on the frontal part of the ear area.

Figure 8 . 
Visualisation of the back ear area was executed with respect to point A. The back component of the bluetooth device was benchmarked against the back ear circumference which was calculated for all 196 headscans as one of the 25 ear dimensions identified during the Basic Benchmarking phase. The 196 curves were overlayed together in the same chart, maintaining a steady, reference point for all curves, which was the Ear Connection Point B, as shown in figure 8 .

Figure 9 .

Point A, linking to the respective point on the product, was identified earlier (see figure 7), as well as points $\mathrm{C}$ (middle point, behind the ear) and $\mathrm{D}$ (lower back ear) were also determined on all 196 headscans. Segments (AB) and (BC) were calculated for all headscans. The geometry of the 196 curves is also available to contribute in the shaping of the back component part (see 4.1.2 in the Findings section).

\subsection{The issuing of focus groups with the help of 3D data analysis methods}

Another meaningful way to apply the 3-dimensional dataset is to generate archetype persons out of the 196 participants, that is, define representative persons of the head and ear geometry with the aim to create a representative user panel to use in the various comfort studies performed during the early and later conceptual phase of the product. The methodology of the generation of the archetypes is described thoroughly in [Stavrakos, Ahmed-Kristensen, 2014]. This section will briefly describe the archetype methodology.

A series of critical ear dimensions were assigned in the issuing of the five product families, as seen in figures 2 and 3. Cluster analysis was performed on the 196 persons in all 5 product categories, selecting for each one out of the five categories, the critical ear and head dimensions associated to each product category. In the example of product category 3 (see figure 3), the three critical ear dimensions were the Concha $\mathrm{X}$, Concha $\mathrm{Y}$ and Inner Circumference. The data was clustered using the Ward's minimum variance method. The 196 participants were clustered based on the three selected ear dimensions. Hence, the data of ears was clustered in 9 meaningful groups. Figure 9 shows an example of how 2 out of the 9 clusters were formed.

Figure 10.

As shown in Figure 9, each cluster contained roughly 15 - 25 participants (in Figure 9, cluster 1 contained 24 persons). In the next step frequency diagrams were made for each ear dimension in each group as shown in Figure 10 for Concha X, Group 1.

Figure 11

This resulted in 3 frequency diagrams for each of the three ear dimensions within each group. Popular intervals were chosen to include at least $60 \%$ of the participants for each group. In the case of Concha $X$ in Figure 10 these intervals were interval 6 and 7. The archetype person was selected based on which person belonged in all three popular intervals for all the three dimensions. The archetypes can then be used by using their scan along for dimensions but can also be used to identify the person for a focus group to collect qualitative feedback. 
This section presents the findings followed by the discussion of findings as derived from the analysis of the collected data which was presented in the $3^{\text {rd }}$ section of this paper. It presents the series of findings from the three main manipulations of the 3D data, i.e., the Basic and InDepth benchmarking between Products and Human Factors, the Visualisation of 3D-Data, and the generation of archetypes using the archetype methodology [Stavrakos \& AhmedKristensen, 2014], in regards to the list of hypothesis presented earlier.

\subsection{Applications of 3-Dimensional Data}

\subsubsection{Basic and In-Depth benchmarking}

This section presents statistical values regarding the critical ear dimensions. Due to confidentiality issues, only part of the findings will be presented. The respective Hypothesis addressed was:

(H1) It is possible to benchmark human dimensions against product dimensions with the aim to support product design.

Figure 11 shows the selected critical ear dimensions and Table 2 shows the respective dimensions retrieved from the head scans regarding the front ear dimensions and the statistical values of MIN, MAX, MEDIAN and AVERAGE values. Concha X ranges from a $\mathrm{MIN}=9,71 \mathrm{~mm}$ to a MAX=27,21 $\mathrm{mm}$ and Concha $\mathrm{Y}$ ranges from $\mathrm{MIN}=11,75 \mathrm{~mm}$ to $\mathrm{MAX}=$ $24,55 \mathrm{~mm}$. For the circumference dimension, the $\mathrm{MIN}=36,26 \mathrm{~mm}$ and the $\mathrm{MAX}=62,04$. These dimensions can inform the product dimensions of the product categories 3, 4, 5 (see figure 2). Especially for in-the-ear headsets the dimensions of the product part which rests inside the ear opening, can be benchmarked against the product dimensions, by consulting Table 2.

Figure 12 .

Table 2.

Table 3 presents the $5^{\text {th }}, 10^{\text {th }}, 20^{\text {th }}, 40^{\text {th }}, 50^{\text {th }}, 70^{\text {th }}, 80^{\text {th }}$ and $90^{\text {th }}$ percentile for the previous dimensions.

Table 3.

The additional dimensions calculated during the In-depth Benchmarking phase (see figure 6 and 7) can be seen in Table 4. These dimensions can contribute in the design of product families 1 and 2, i.e., the design of headphones over and around the ear; for example, the head height can be benchmarked against the height of the headband of the headphones.

Table 4. 
The knowledge of the angles $\varphi 1$ and $\varphi 2$ can be used to define the degree of fluctuation of the ear cups of the headphones along different axis as well as contribute in the determination of the pressure levels that the ear cups of the headphones exercise on the head. These findings prove the first hypothesis $\mathrm{H} 1$, that product dimensions can indeed be informed by human dimensions. The effect of these manipulations on the various phases of early conceptual design will be discussed in the next session.

\subsubsection{Visualisations of Data}

The Hypothesis for these findings was: $(\mathrm{H} 2)$ It is possible to predict product dimensions through qualitative methods of visualisation of human data. Table 5 contains statistical values linking to the segments identified during the phase of Visualisation of 3D data (see figure 8).

Table 5.

Figure13.

The table of percentiles is presented here (See Table 6).

Table6.

The human dimensions presented have been associated with product dimensions (see figure 12). These can satisfy the demand for dimensioning of more complicated product parts in terms of shape and placement in the product geometry. These findings prove the second hypothesis. The potential of this method as well as more options for supporting early conceptual design will be discussed in the next (discussion) session.

\subsection{Discussion and Implications}

This section presents a discussion regarding the phases of the methodological framework and other aspects regarding the applications of 3-dimensional data which were presented earlier during the Findings section.

\subsubsection{Regarding the methodological framework}

In the Early Product Understanding phase the critical dimensions are defined and they are associated to respective human data. The product categories were defined based on fit. In this phase, it was advisable to use a high number of dimensions in order to cover as much of the complexity of the products as possible. In the case that product components interacted with different body areas, a matrix of product components versus critical dimensions (instead of product categories versus human dimensions) was created to make more direct associations between human data and products.

For the collection of 3-dimensional data, a homogenous dataset, in terms of origin of the population at hand, led to a representative sample, like in the example of the Danish population, used in this paper. 
The various Applications of 3-Dimensional data were concerned with different phases of the design process. The applications of the data are discussed here. Benchmarking between product and human factors, where human data informs basic product dimensions, could be utilised in the early prototyping phase where basic issues of shaping and contour are still at stake. The in-depth benchmarking can be used in the refinement of prototypes, where mechatronic components are added into the prototype. In this phase, issues of slight alterations in the dimensioning of the prototype may arise, to fit the various components inside the basic prototype casing. The visualisation of data can contribute both in early and later stages of the design process, as the method can provide defining dimensions of the basic prototype (e.g. the head circumference dimension, which is measured from top ear to top ear, over the head, informs the length of the headband of a headphone product) as well as give an impression of more complicated dimensions, the need for which is high in later stages of the product development (e.g. segments $(\mathrm{AB})$ and $(\mathrm{BC})$ in the example provided in the presentation of Visualisations of data in section 3.5).

\subsubsection{The creation of focus groups}

The creation of focus groups with the utilisation of the archetype methodology is contributing primarily to the execution of more reliable comfort studies. Once the archetypes have been defined these can be used in two ways. The first way is to use the archetype's dimensions in order to design comfort studies where the researcher could make inquiries on physical properties of comfort towards new prototypes. However, it is important that the prototypes have similar attributes (e.g. similar manner of use, similar geometry, etc) to the product used at the beginning of the method in order to ensure the validity of the archetypes.

The second way is to define test panels based on the generated archetypes. These panels can be used for both quantitative and qualitative studies. The method adopts the approach of identifying an archetype from clusters analysis on a large set of data, which can then be used to identify participants for a test panel rather than including a large number of participants in user studies of comfort.

\subsubsection{Limitations of the study}

In this paper, each product was seen as a whole, homogenous object due to product complexity and time limitations. The segmentation of the product into components will create various interfaces with different physical properties and reveal additional cases of critical dimensions and good fit. This study was limited to one product type, that is, external-earworn products. The application of the methods on more product types will showcase additional issues of physical comfort and this will offer new knowledge in the comfort theory and methodology. Regarding the collection of 3D data; this was limited to head physiology, as this is the only area of interest for the case study presented. Full body scans will offer the possibility to include additional body worn product types. Moreover, enriching the data with more scans will increase the dataset's reliability in terms of accuracy of the presented dimensions. Regarding the selection of reference points on the 3D head scans; these were identified manually on the various heads, with the help of the 3D software, which adds an experimental error in the calculation of the anthropometric dimensions. Regarding the visualisation of the data by overlaying the various curves, the assessment for each dimension 
requires a series of manual management in the 3D software, which is time consuming. The use of image software or other software with integrated functions for superimposing geometric curves would save time in the execution of the method. Limitations for the archetype methodology can be found in [Stavrakos, Ahmed-Kristensen, 2014)].

\section{Conclusion}

In this paper, the incorporation of anthropometry in the design of products was examined. This research utilised the example of a case study from the industry of external-ear worn products. The collection of anthropometric data was presented, however, the major contributions of this paper concern the various applications of the data which involve (1) the benchmarking between human and product data, (2) the visualisations of the data and (3) the forming of archetypes based on the collected dataset.

These applications led to an increased understanding of human and product anatomy (providing 29 basic and 9 advanced human dimensions from a geometrical point of view). A methodological framework was provided to ensure the reproducibility of the methods. In addition to the methodological framework presented in this paper, these methods are expected to expand to other industries of body - worn products, such as helmets, etc., with the acquirement of respective human data.

These methods are expected to assist designers at various stages of the design process by contributing to an increased understanding of human geometry and product complexity, achieving a more accurate benchmarking between humans and products and leading to the execution of more reliable comfort studies.

\section{Acknowledgements}

The authors would like to thank Tomasz Goldman and Charles Pedersen for their supervision throughout the project and the 196 participants for their participation in the scanning sessions. 


\section{Reference List}

Ghoddousi, H., R. Edler, et al., "Comparison of three methods of facial measurement." International Journal of Oral and Maxillofacial Surgery, Vol. 36 (3) 2007. Pp. 250-258.

Hennessy, R. J., A. Kinsella, et al., "3D laser surface scanning and geometric morphometric analysis of craniofacial shape as an index of cerebro-craniofacial morphogenesis: initial application to sexual dimorphism", Biological Psychiatry, Vol. 51(6), 2007. Pp. 507-514.

Hibaru and Watanabe, "A procedure for allocating chairs to school children", Hard facts about soft machines, London, 1994

Jung Hwa S., Hyung-Shik Jung, "Surveying the dimensions and characteristics of Korean ears for the ergonomic design of ear-related products", Received, 23 May 2001

Kee, D. and I. Lee "Relationships between subjective and objective measures in assessing postural stresses", Applied Ergonomics, Vol 43 (2), 2012, pp. 277-282.

Klamklaya, , Angoon Sungkhaponga, Nantakrit Yodpijitb, Patrick E. Pattersonc, "Anthropometry of the southern Thai population", International Journal of Industrial Ergonomics, Vol. 38(1), 2008, pp. 111-118

Parcells et al., 1999, C. Parcells, M. Stommel, R.P. Hubbard, "Mismatch of classroom furniture and student body dimensions: empirical findings and health implications", Health, Vol. 24 (4), 1999, pp. 265-273

Peoplesize Anthropometric Database, http://www.openerg.com/psz/anthropometry_data.html, 2008

Richards, L. G. "On the psychology of passenger comfort", Human Factors in Transport Research, Vol. 2: User Factors: Comfort, The Environment and Behaviour, 1980, pp 15-23

Sanders and McCormick, "Human Factors in Engineering and Design", (seventh ed), M.S. Sanders and E.J. McCormick, McGraw-Hill, New York, 1993

Slater K. "Human Comfort (Book)." Contemporary Sociology 16(6), 1987

Stavrakos, S-K \& Ahmed-Kristensen, Goldman, T, 2014, "Using archetypes to create user panels for usability studies" in Design 2014 - International Design Conference. Design Society, pp. 2157-2166

Stavrakos, S-K \& Ahmed-Kristensen, 2012, "Assesment of anthropometric methods in headset design", in Design 2012 - International Design Conference. Design Society, pp. 1123-1132.

Vink, P., "Comfort and design: principles and good practice", Boca Raton CRC Pres, 2005

Weinberg, Naidoo, Govier, Martin, Kane, Marazita, "Anthropometric Precision and Accuracy of Digital ThreeDimensional Photogrammetry" Journal of Craniofacial Surgery, Vol. 17(3), 2006, pp. 477-483

Zenk, R., M. Franz, et al. "Technical note: Spine loading in automotive seating." Applied Ergonomics 43 (2): 290-295. 


\section{Tables}

Table 1. Demographics of the participants and account of performed measurements

\begin{tabular}{|l|l|l|l|}
\hline & Men & Women & Total \\
\hline Number & 98 & 98 & 196 \\
\hline Nationality & Danish & Danish & \\
\hline $\begin{array}{l}\text { Human Dimensions measured on } \\
\text { both ears }\end{array}$ & $25 \times 196$ & 4.900 \\
\hline $\begin{array}{l}\text { Advanced Human Dimensions } \\
\text { measured on both ears }\end{array}$ & $9 \times 196$ & 1.764 \\
\hline Total & & $\mathbf{6 , 6 6 4}$ measurements \\
\hline
\end{tabular}

Table 2. Statistical Values for front ear dimensions

\begin{tabular}{|c|c|c|c|c|}
\hline & \multicolumn{4}{|c|}{ Statistical Values (mm) } \\
\hline & MIN & MAX & MEDIAN & AVERAGE \\
\hline Ear Height & 9,71 & 28,78 & 19,47 & 51,11 \\
\hline Concha X & 13,27 & 27,21 & 19,31 & 17,74 \\
\hline Concha Y & 11,75 & 24,55 & 17,88 & 19,45 \\
\hline Circumference & 36,26 & 62,04 & 50,44 & 93,11 \\
\hline
\end{tabular}

Table 3. Percentiles for front ear dimensions

\begin{tabular}{|c|c|c|c|c|c|c|c|c|}
\hline \multirow{2}{*}{ Ear dimensions } & \multicolumn{7}{|c|}{ Table of percentiles (mm) } \\
\cline { 2 - 10 } & $\mathbf{5 \%}$ & $\mathbf{1 0 \%}$ & $\mathbf{2 0 \%}$ & $\mathbf{4 0 \%}$ & $\mathbf{5 0 \%}$ & $\mathbf{7 0 \%}$ & $\mathbf{8 0 \%}$ & $\mathbf{9 0 \%}$ \\
\hline \multirow{2}{*}{ Ear height } & 14,00 & 15,16 & 16,63 & 18,37 & 19,47 & 20,86 & 22,26 & 23,70 \\
\hline Concha X & 15,95 & 16,49 & 17,42 & 18,60 & 19,34 & 20,78 & 21,32 & 22,65 \\
\hline Concha Y & 14,61 & 15,03 & 15,88 & 17,32 & 17,89 & 18,80 & 19,52 & 20,41 \\
\hline Circumference & 43,39 & 44,84 & 47,46 & 49,35 & 50,37 & 53,60 & 55,32 & 57,38 \\
\hline
\end{tabular}

Table 4. Statistical Values for head dimensions and angles $\varphi 1, \varphi 2$

\begin{tabular}{|c|c|c|c|c|}
\hline & \multicolumn{4}{|c|}{ Statistical Values } \\
\hline & MIN & MAX & MEDIAN & AVERAGE \\
\hline Head breadth Top & 135,76 & 175,26 & 154,51 & 135,76 \\
\hline Head Breadth Bottom & 111,76 & 173,54 & 140,22 & 111,76 \\
\hline Head Height & 11,77 & 163,18 & 124,74 & 11,77 \\
\hline $\boldsymbol{~} 1$ & 0,28 & 40,23 & 19,22 & 0,28 \\
\hline $\boldsymbol{\varphi 2}$ & 7,74 & 26,49 & 154,48 & 7,74 \\
\hline
\end{tabular}


Table 5. Statistical Values for segments (AB) and (BC)

\begin{tabular}{|c|c|c|c|c|}
\hline & \multicolumn{4}{|c|}{ Statistical Values } \\
\hline & MIN & MAX & MEDIAN & AVERAGE \\
\hline (AB) & 10,71 & 29,78 & 19,72 & 19,94 \\
\hline (BC) & 7,21 & 22,98 & 13,93 & 14,20 \\
\hline
\end{tabular}

Table 6. Percentiles for segments (AB) and (BC)

\begin{tabular}{|r|c|c|c|c|c|c|c|c|}
\hline & \multicolumn{10}{|c|}{ Table of percentiles } \\
\hline & $\mathbf{5 \%}$ & $\mathbf{1 0 \%}$ & $\mathbf{2 0 \%}$ & $\mathbf{4 0 \%}$ & $\mathbf{5 0 \%}$ & $\mathbf{7 0 \%}$ & $\mathbf{8 0 \%}$ & $\mathbf{9 0 \%}$ \\
\hline Segment (AB) & 14,72 & 15,91 & 16,90 & 18,77 & 19,72 & 21,47 & 22,57 & 24,33 \\
\hline Segment (BC) & 8,11 & 8,11 & 10,01 & 11,89 & 12,43 & 14,11 & 15,11 & 16,69 \\
\hline
\end{tabular}




\section{Figure Captions}

Figure 1. Methodological framework of 3D manipulations

Figure 2. The 5 product families of external-ear worn products

Figure 3. Matrix of associations for product categories 1 and 3

Figure 4. Scanning and Refinement of Scans

Figure 5. Generation of critical human dimensions

Figure 6. Presentation of critical points and additional ear and head dimensions

Figure 7. Presentation of angles $\varphi 1$ and $\varphi 2$

Figure 8. Mechanical model of a product worn behind the ear

Figure 9. Mechanical model of a product worn behind the ear

Figure 10. Part of the 9 groups - clusters as derived from Ward's method of minimum variance

Figure 11. Frequency diagrams for concha X, Group 1

Figure 12. Front ear dimensions

Figure13. Benchmarking of segments (AB) and (BC) 


\section{Figures}

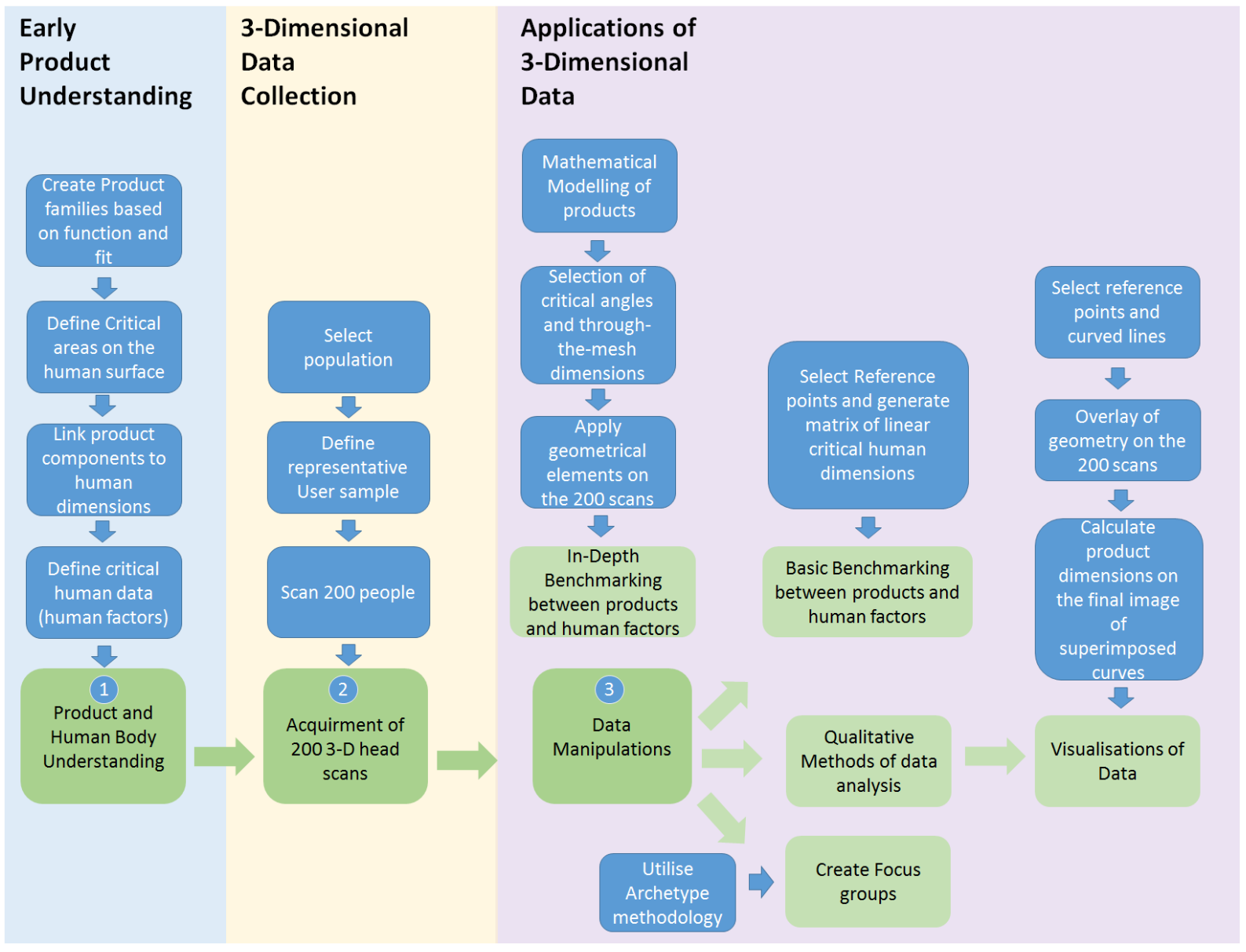

Figure 1. Methodological framework of 3D manipulations

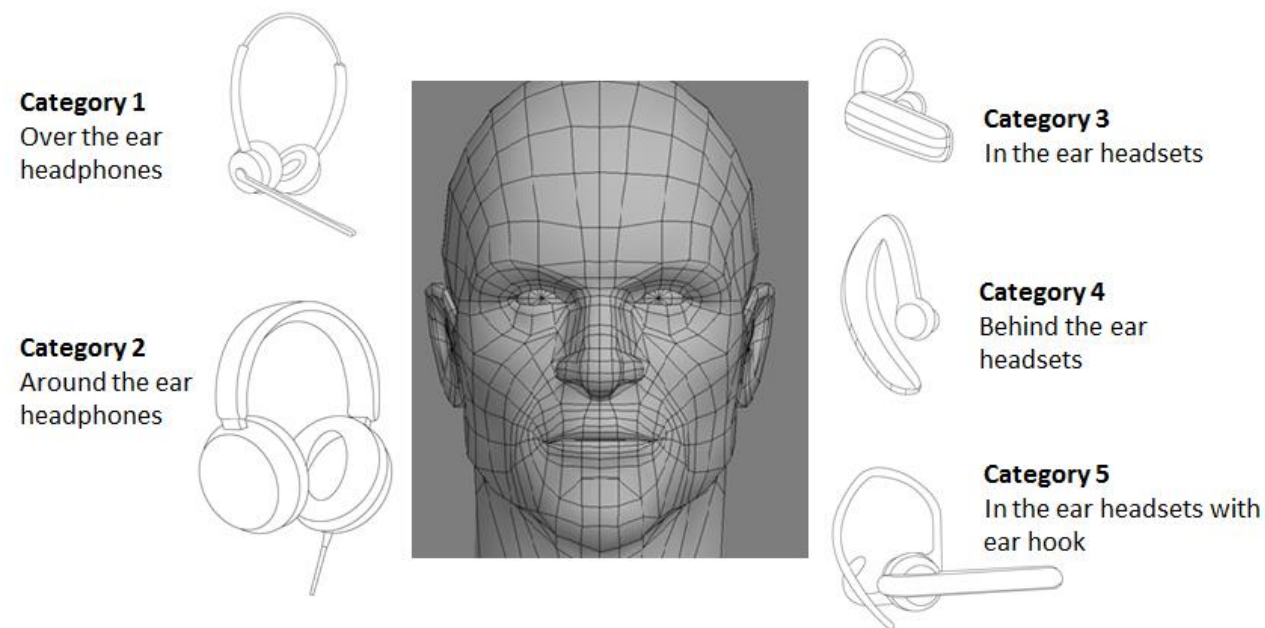

Figure 2. The 5 product families of external-ear worn products 


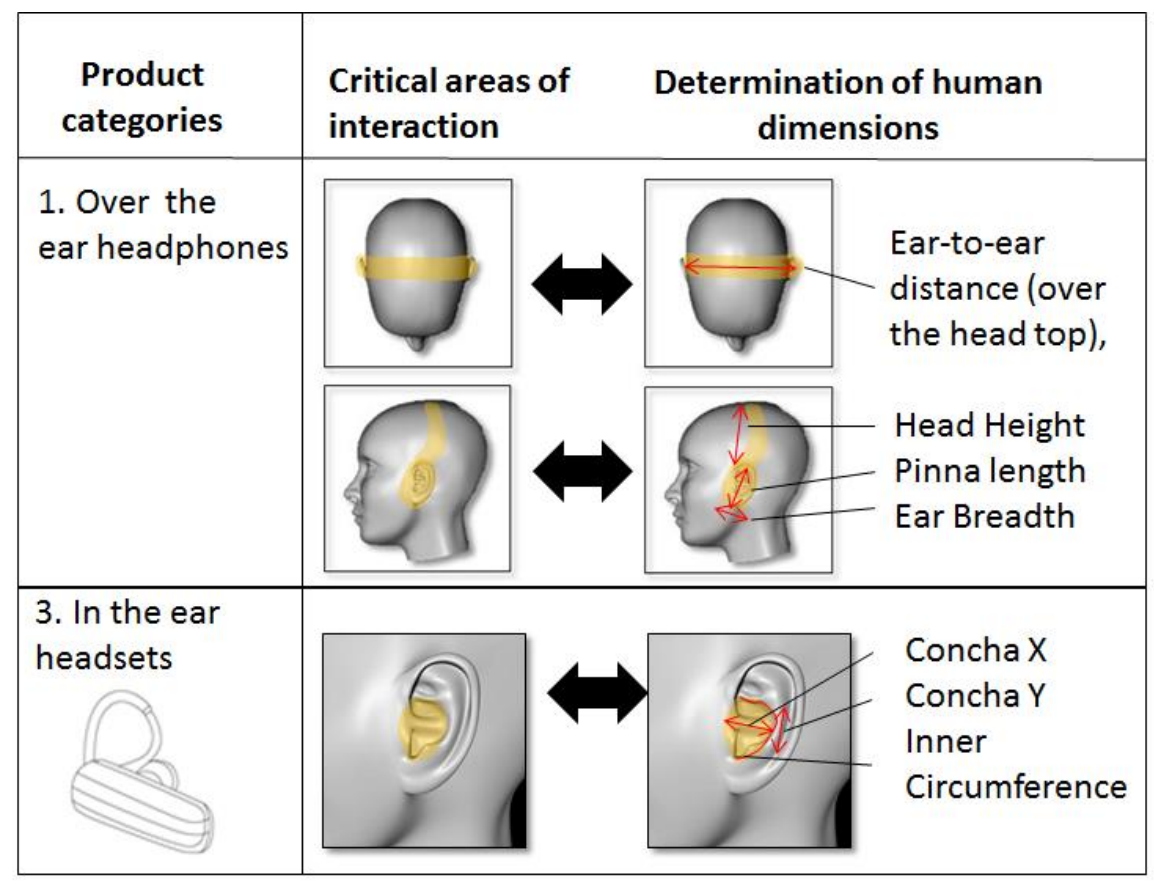

Figure 3. Matrix of associations for product categories 1 and 3

\section{3-D Scanning}

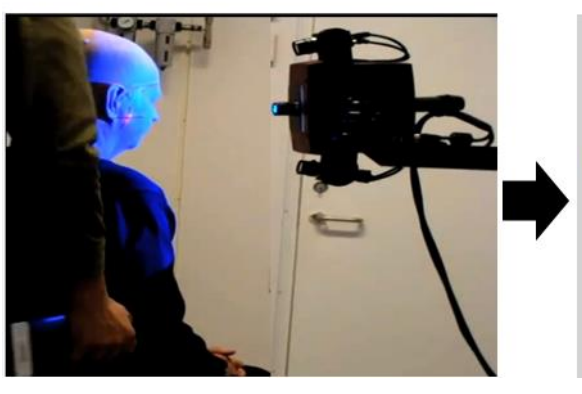

Refinement of Scans

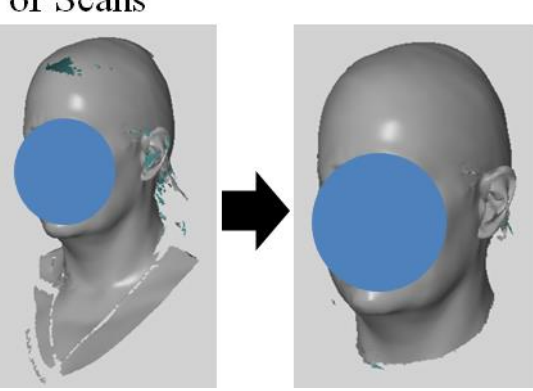

Figure 4. Scanning and Refinement of Scans

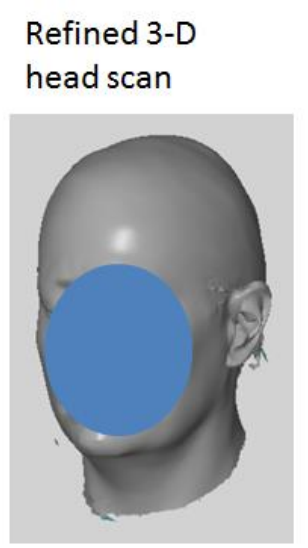

\begin{abstract}
Application of Reference points on the $3 D$ scan
\end{abstract}

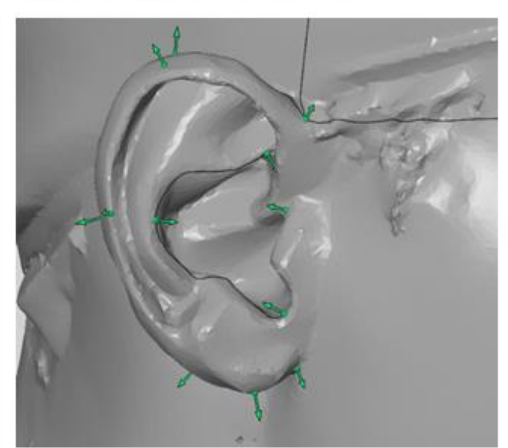

Generation of critical dimensions

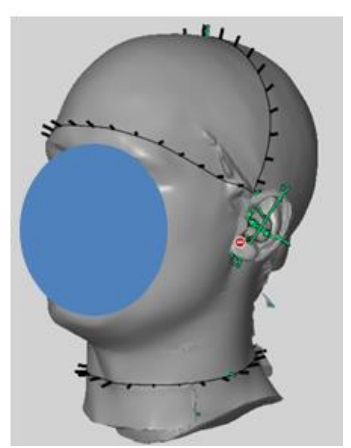

Figure 5. Generation of critical human dimensions 

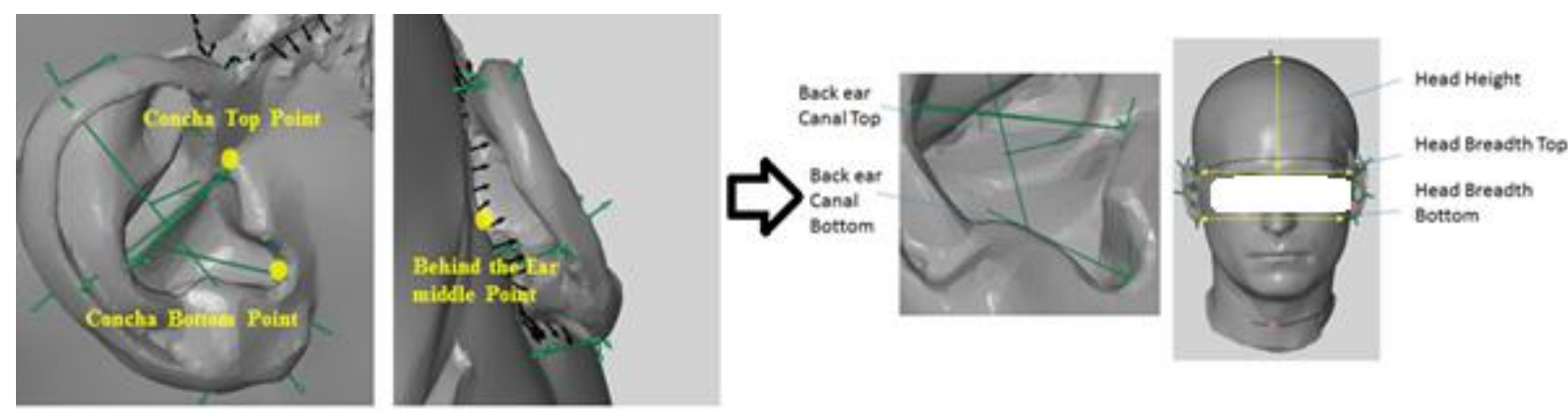

Figure 6. Presentation of critical points and additional ear and head dimensions

\section{On the Mesh}

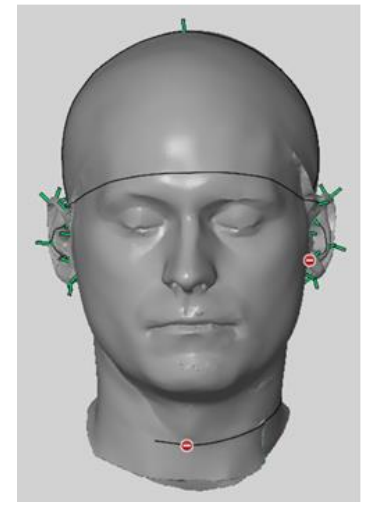

Calculation of angles $\phi 1$ and $\phi 2$ through the mesh

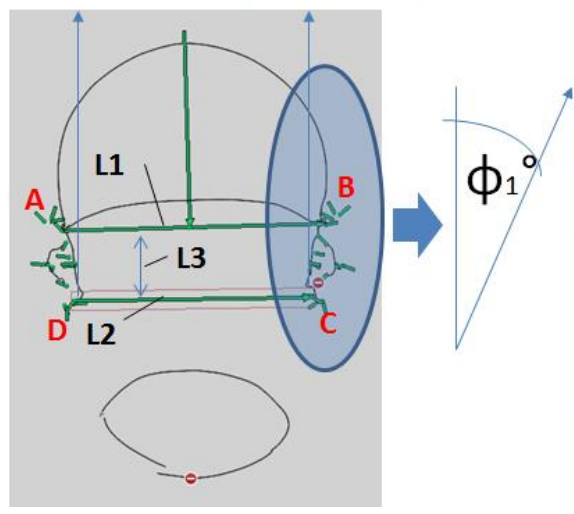

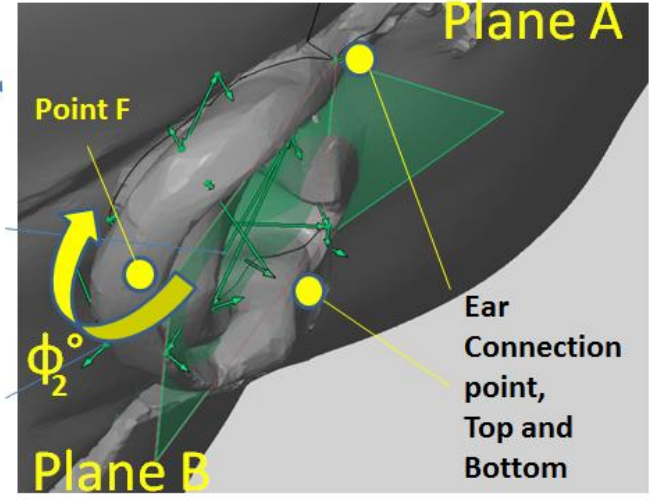

Figure 7. Presentation of angles $\varphi 1$ and $\varphi 2$

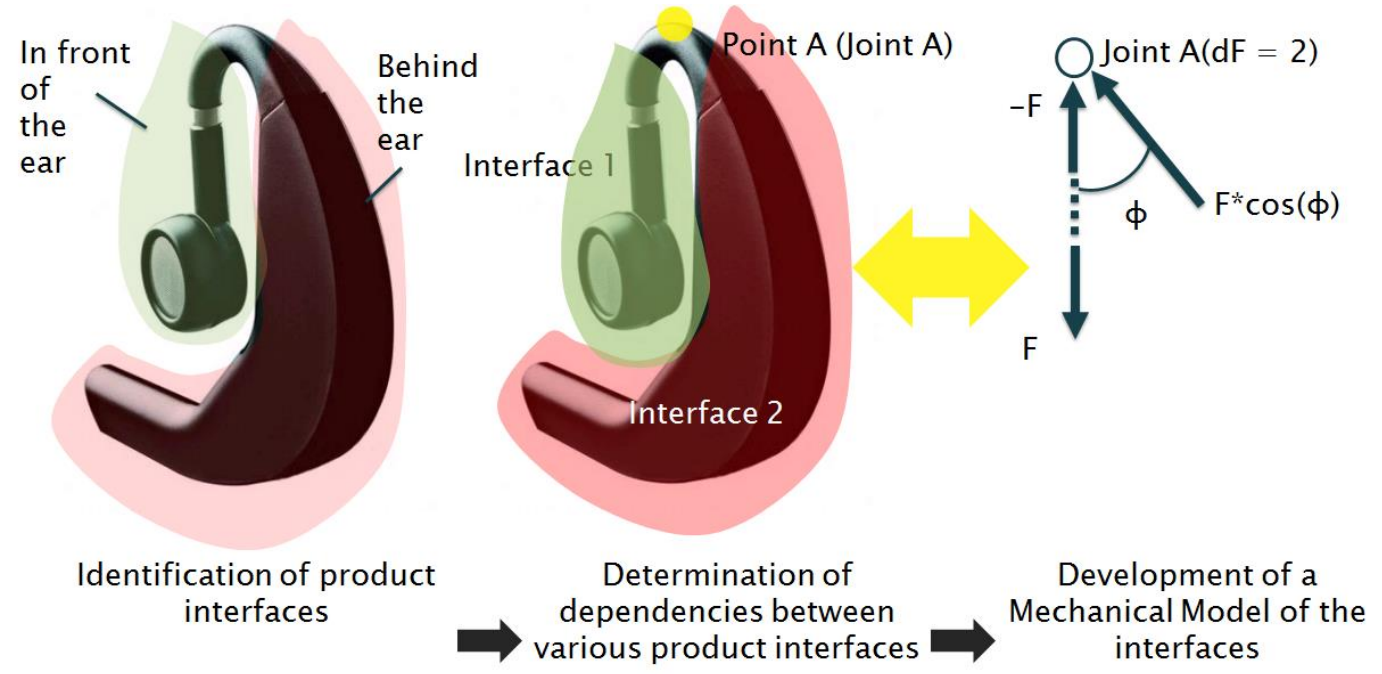

Figure 8. Mechanical model of a product worn behind the ear 

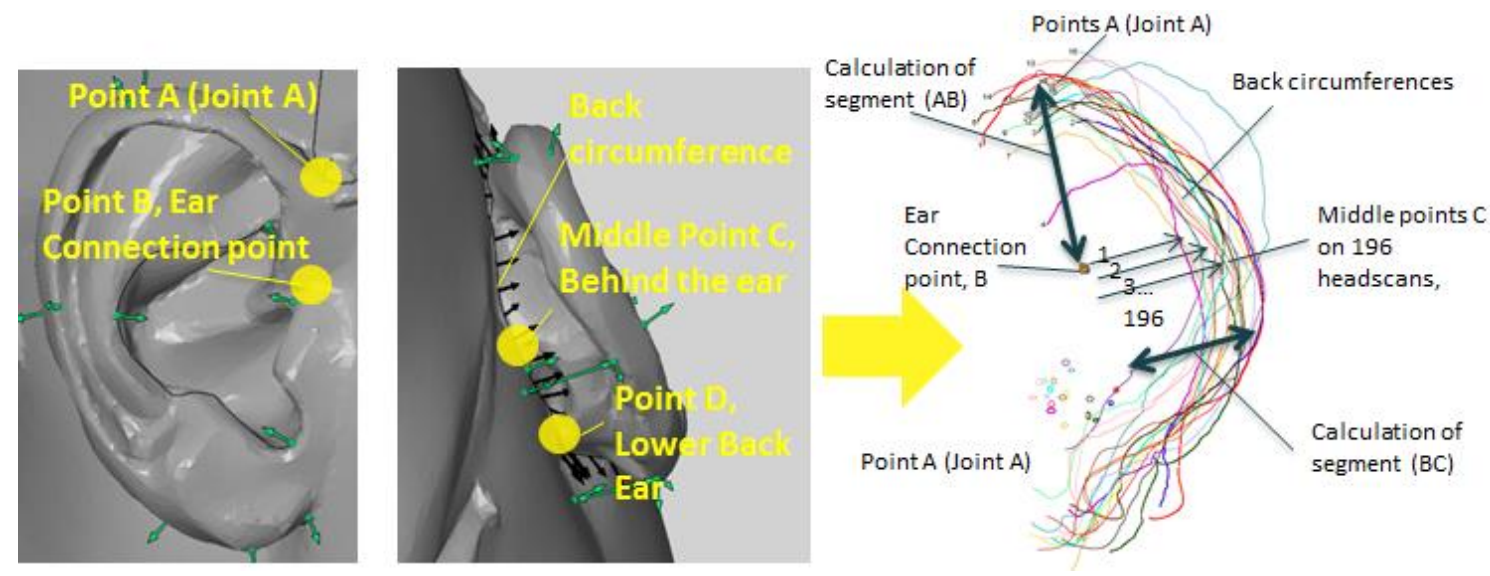

Figure 9. Mechanical model of a product worn behind the ear

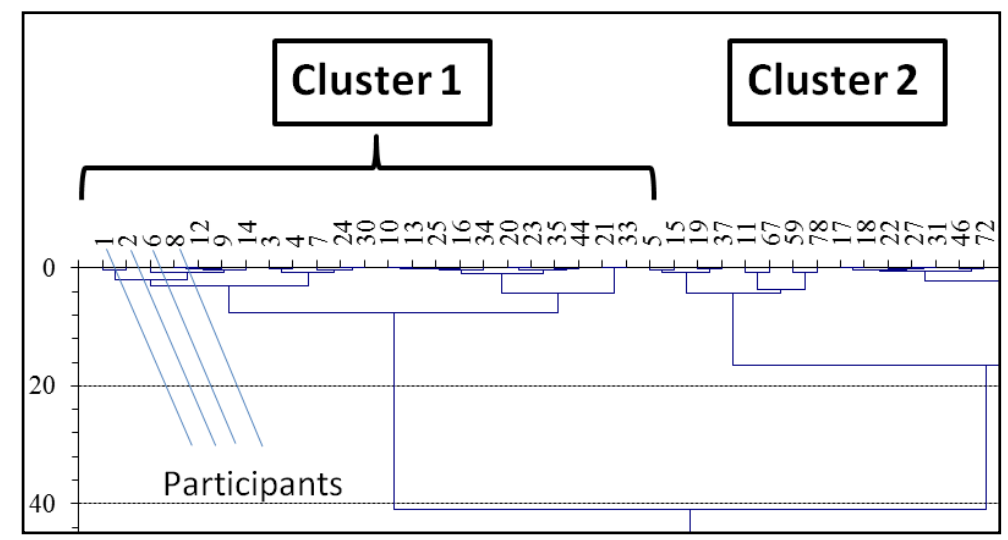

Figure 10. Part of the 9 groups - clusters as derived from Ward's method of minimum variance

\begin{tabular}{|c|c|c|c|c|}
\hline & & \multicolumn{3}{|c|}{ Bin Limits } \\
\hline MIN & 12,27 & 0 & 12,27 & 1 \\
\hline MAX & 17,61 & 1 & 13,03 & 0 \\
\hline AVG & 15,85 & 2 & 13,80 & 0 \\
\hline & 0,763 & 3 & 14,56 & 2 \\
\hline & & 4 & 15,32 & 2 \\
\hline & & 5 & 16,08 & 1 \\
\hline & & 6 & 16,85 & 7 \\
\hline & & 7 & 17,61 & 3 \\
\hline
\end{tabular}

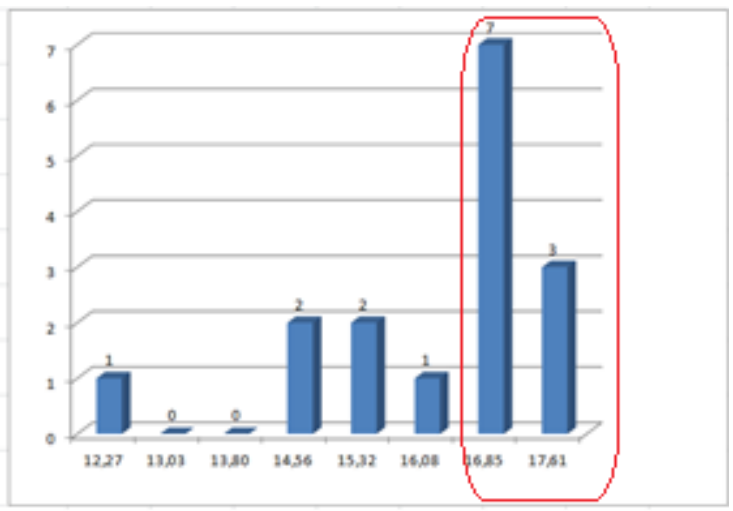

Figure 11. Frequency diagrams for concha X, Group 1 


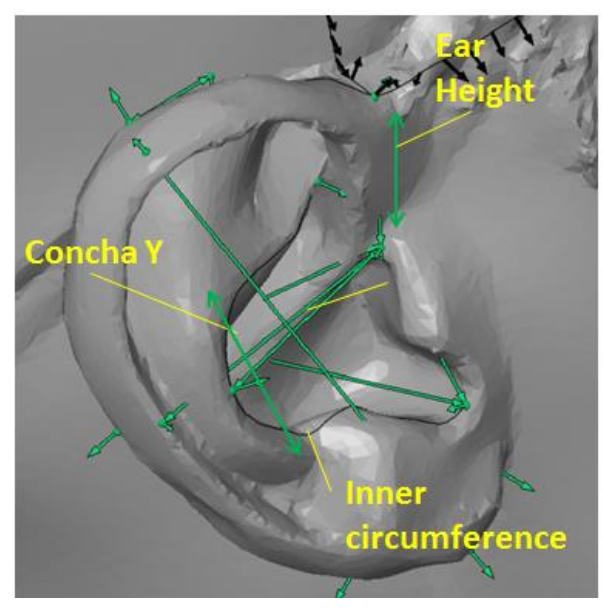

Figure 12. Front ear dimensions

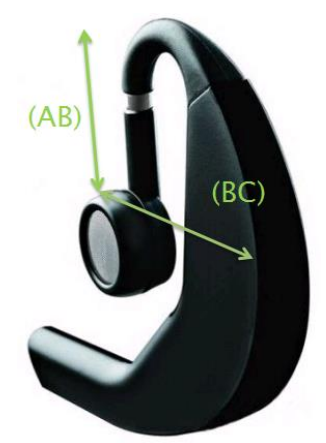

Figure13. Benchmarking of segments (AB) and (BC) 